\title{
The effects of radiofrequency on the bacteriological and histological characteristics of tonsils in patients with chronic and persistent tonsillitis
}

\author{
Shadman Nemati ${ }^{\mathrm{a}, *}$, Fahimeh Mohammadghasemi ${ }^{\mathrm{b}}$, Ali Mojtahedi ${ }^{\mathrm{b}}$, Ali Faghih Habibi ${ }^{\mathrm{a}}$, \\ Samaneh Rouhi ${ }^{\mathrm{c}}$, Ehsan Kazem Nejad Leili ${ }^{\mathrm{d}}$, Mahboobeh Moroosi ${ }^{\mathrm{a}}$ \\ a Otorhinolaryngology Research Center, Department of Otolaryngology and Head and Neck Surgery, School of Medicine, Guilan University of Medical Sciences, Rasht, Iran \\ ${ }^{\mathrm{b}}$ Cellular and Molecular Research Center, School of Medicine, Guilan University of Medical Sciences, Rasht, Iran \\ ${ }^{c}$ Medical Microbiology Research Center, Qazvin University of Medical Sciences, Qazvin, Iran \\ ${ }^{\mathrm{d}}$ Guilan Road Trauma Research Center, Guilan University of Medical Sciences, Rasht, Iran
}

\section{A R T I C L E I N F O}

\section{Keywords:}

Radiofrequency

Tonsil

Histology

Nodule

Morphometry

Bacteriology

Colony

S. aureus

\begin{abstract}
A B S T R A C T
Objectives: Tonsillotomy with radiofrequency (RF) is one of the newest treatments for chronic tonsillitis, but the mechanism of RF effects and complications are still pending. The aim of this study was to evaluate the effects of RF on the histological and bacteriological characteristics of the tonsils (Case-control study).

Materials and methods: In fifty-two patients with chronic tonsillitis in 2017-2018, immediately after tonsillectomy, the tonsils were divided into 2sections; one sample treated with RF, and the other one considered as control, without intervention. All tonsil samples sent for histological and bacteriological study: morphometric assays made by Digitizer software, and type of bacterial colonies identified by microbiological and biochemical tests. Willcoxon and McNemar tests were used for statistical analysis and level of significance was $p \leq .05$.

Results: Tonsil mucosal thickness (2202.98 \pm 323.09 vs. $2463.94 \pm 357.61 \mu \mathrm{m})$ and size of the tonsil nodule $\left(28,000.42 \pm 9608.75\right.$ vs. $\left.36,692.81 \pm 7040.74 \mu \mathrm{m}^{2}\right)$ were significantly lower in the RF + group than other group ( $p=.001$ and $p=.01$, respectively). There was no significant differences in thickness of the tonsil epithelium $(p=.075)$, number of lymphoid nodules $(p=.860)$, and the number of reticular tonsil epithelium ( $p=.813$ ) between the two groups. Bacterial growth in RF- and RF + groups had no statistically significant difference $(p=.06)$, however, the average colony count of $S$. aureus in RF + tonsils were significantly lower, and total number of bacterial colonies were significantly lower in RF $+\operatorname{group}(1405 \pm 156$ vs. $2471 \pm 156)$, $(p=.001)$.

Conclusion: RF surgery has significant effects on size of the nodules, thickness of the mucous layer and bacteriological characteristics of tonsil tissue. Especially $S$. aureus seems to be more sensitive to RF effects.
\end{abstract}

\section{Introduction}

Tonsillitis is described as inflammation of the pharyngeal tonsils [1]. Bacteria such as Haemophilus influenza, Streptococcus pneumonia, viridans group Streptococci, Staphylococcus epidermidis, Staphylococcus aureus, Chlamydophila pneumonia and Mycoplasma pneumonia, and many viruses such as respiratory syncytial viruses (RSV) a and b, influenza virus $A$ and $B$ (IVA, IVB), human Parainfluenza viruses 1, 2, and 3 (HPIVS), human adenoviruses (HADS), are common pathogens [2,3]. Typical symptoms of tonsillitis are sore throat, swallowing discomfort, painful lymph nodes in the neck; fever over $38{ }^{\circ} \mathrm{C}$, headache, loss of appetite and bad breath. It may cause peritonsillaror quinsy abscess [2].
Epidemiological studies show that more tonsillitis cases are seen in age group 6-12 years-old with 69\% frequency [4]. Treatment of tonsillitis is possible using ibuprofen or acetaminophen with or without antibiotics. Surgical treatment that named tonsillectomy is also considered for patients with resistant and chronic tonsillitis [2]. Tonsillectomy is one of the most commonly performed surgical procedures worldwide $[5,6]$. In a report, $36.8 \%$ of tonsillectomy patients were younger than 12 yearsold and $63.2 \%$ were over 12 years-old; $39.3 \%$ was male and $60.7 \%$ was female [7]. Also in UK Incidence of tonsillectomy was 4.2 per 1000 person in years [8]. There are different tonsillectomy techniques; including cold-knife tonsillectomy, monopolar and bipolar diathermy, cryosurgery, suction diathermy, bipolarscissor, KTP-532 laser, CO2

\footnotetext{
*Corresponding author at: Otorhinolaryngology Research Center, Department of Otolaryngology and Head and Neck Surgery, School of Medicine, Guilan University of Medical Sciences, Amir Al-Momenin Hospital, Rasht, Iran.

E-mail address: drshadmannemati_ent@yahoo.com (S. Nemati).
} 